\title{
Parâmetros Climáticos e Incremento Diamétrico de Espécies Florestais em Floresta Aluvial no Sul do Brasil
}

\author{
Maria Raquel Kanieski ${ }^{1}$, Franklin Galvão², Tomaz Longhi-Santos², \\ Jaçanan Eloísa de Freitas Milani², Paulo Cesar Botosso ${ }^{3}$ \\ ${ }^{1}$ Engenharia Florestal, Universidade do Estado de Santa Catarina - UDESC, Lages/SC, Brasil \\ ${ }^{2}$ Departamento de Ciências Florestais, Universidade Federal do Paraná - UFPR, Curitiba/PR, Brasil \\ ${ }^{3}$ Embrapa Florestas, Colombo/PR, Brasil
}

\begin{abstract}
RESUMO
Objetiva-se com este estudo avaliar a periodicidade do incremento em diâmetro do tronco de Sebastiania commersoniana e das espécies arbóreas exóticas invasoras Hovenia dulcis e Ligustrum lucidum em remanescente de Floresta Ombrófila Mista Aluvial em Araucária, Paraná. Foram selecionados indivíduos presentes no dossel e sub-bosque. Cintas dendrométricas foram instaladas em todos os indivíduos selecionados, que foram monitorados mensalmente, de julho de 2009 a junho de 2012. Os incrementos correntes mensais e anuais foram correlacionados com precipitação e temperatura. As espécies exóticas e os indivíduos do dossel apresentaram incremento superior à espécie nativa, que teve pouca variação ao longo do ano, e aos indivíduos de sub-bosque. Correlações significativas do incremento foram obtidas com temperatura e precipitação, mostrando que o crescimento em diâmetro está, em grande parte, relacionado às condições meteorológicas.
\end{abstract}

Palavras-chave: cinta dendrométrica, Floresta Ombrófila Mista, espécie invasora.

\section{Climatic Parameters and Diameter Increment of Forest Species in Alluvial Forest in Southern Brazil}

\begin{abstract}
This study aims to evaluate the periodicity of trunk diameter increment of Sebastiania commersoniana and the two invasive exotic tree species, Hovenia dulcis and Ligustrum lucidum, in a remnant area of Alluvial Mixed Rain Forest at Araucaria, Paraná. We selected individuals of the different species present from canopy and understory. Dendrometric bands were installed in all individuals, which were monitored monthly, from July 2009 to June 2012. The monthly and annual current increments were correlated with precipitation and temperature. Exotic species and canopy individuals presented increment higher than native species, which had low variation in the year, and understory individuals. Significant correlations were obtained between the increment diameter and temperature and precipitation, showing diameter growth of the species is mostly conditioned by the meteorological conditions.
\end{abstract}

Keywords: dendrometric bands, Mixed Rain Forest, invasive species. 


\section{INTRODUÇÃO}

As florestas aluviais, também chamadas de florestas de galeria ou ciliares, exercem importante função na estabilidade do regime hídrico das planícies sujeitas a inundações periódicas, decorrentes do transbordamento dos rios em ocasiões de máximas pluviométricas (Socher et al., 2008).

O remanescente de floresta aluvial no âmbito de Floresta Ombrófila Mista existente na área da Refinaria Presidente Getúlio Vargas (REPAR), unidade da Petróleo Brasileiro S.A. (Petrobras), no município de Araucária, $\mathrm{PR}$, banhado pelo rio Barigui, sofre as consequências da larga ocupação urbana ao seu redor, bem como do vazamento de quatro milhões de litros de óleo bruto que atingiu essa área no ano de 2000. Não bastasse a pressão antrópica, as espécies nativas nos remanescentes florestais existentes estão progressivamente perdendo espaço por meio da competição com espécies exóticas que estão se estabelecendo no local, comprometendo também a função ecossistêmica no ambiente.

Quando introduzidas em locais onde não ocorrem naturalmente, as espécies exóticas, na maioria das vezes, não encontram competidores ou predadores e, consequentemente, têm sua ocupação e multiplicação facilitada, o que acaba ameaçando a permanência de espécies nativas, principalmente em ambientes degradados (Rodolfo et al., 2008). Segundo a Portaria n. 95/2007 do IAP (Paraná, 2007), as espécies Hovenia dulcis Thunb. e Ligustrum lucidum W. T. Aiton. são classificadas como exóticas invasoras para o estado do Paraná. Esse fator pode ser uma ameaça a mais para as espécies nativas existentes na área de estudo, e que traz a necessidade de um maior conhecimento sobre os padrões de desenvolvimento e interação das diferentes espécies exóticas e nativas, assim como obtenção de informações que subsidiem práticas de manejo com vistas à conservação dessas áreas.

$\mathrm{O}$ crescimento das árvores é influenciado pelas características da espécie interagindo com o ambiente (Husch et al., 1982). Inúmeros trabalhos vêm sendo realizados para avaliar a influência das variáveis ambientais no crescimento de espécies tropicais e subtropicais, principalmente variáveis meteorológicas (e.g.: Andreacci et al., 2014; Brienen \& Zuidema, 2006; Canetti et al., 2014; Ferreira-Fedele et al., 2004; Kanieski et al., 2012, 2013; Lisi et al., 2008; Longhi-Santos,
2013; Nutto \& Watzlawick, 2002; Spathelf et al., 2000), sendo que precipitação, temperatura e disponibilidade de água no solo seriam, dentre os fatores observados, os que mais influenciam no crescimento.

Tendo em vista a grande pressão no remanescente de Floresta Ombrófila Mista Aluvial em Araucária, PR, e a sua importância para manutenção das funções ecológicas no local, este estudo objetiva avaliar o incremento em diâmetro do tronco da espécie nativa de maior representatividade na área, o branquilho [Sebastiania commersoniana (Baill.) L. B. Sm. e Downs - Euphorbiaceae] e das espécies exóticas invasoras uva-do-Japão (Hovenia dulcis - Rhamnaceae) e alfeneiro (Ligustrum lucidum - Oleaceae), considerando a posição sociológica que os indivíduos ocupam e procurando correlacionar o crescimento com as variáveis meteorológicas de precipitação e temperatura.

Espera-se que haja crescimento diferenciado entre as espécies analisadas, entre os indivíduos do dossel e sub-bosque, e que o crescimento em diâmetro esteja atrelado às variáveis meteorológicas estudadas.

\section{MATERIAL E MÉTODOS}

A área de estudo está localizada no município de Araucária, região metropolitana de Curitiba, $\mathrm{PR}$, próximo às coordenadas $25^{\circ} 34^{\prime} 02^{\prime \prime} \mathrm{S}$ e $49^{\circ} 20^{\prime} 53^{\prime \prime} \mathrm{W}$, em uma altitude média de $890 \mathrm{~m}$ s.n.m. O fragmento florestal está estabelecido em uma planície inundável do rio Barigui, principal tributário da margem direita do rio Iguaçu, no primeiro planalto paranaense, onde os solos hidromórficos são predominantemente dos tipos Gleissolos Háplicos e Melânicos. Condição essa muito particular quando comparada às demais formações da Floresta Ombrófila Mista de encosta.

O clima é do tipo Cfb (Köppen), com chuvas bem distribuídas durante o ano, com inverno rigoroso e geadas frequentes. A média de precipitação para a região, considerando-se série histórica de 1961-2011, é de $1.490 \mathrm{~mm} / \mathrm{ano}$, sendo o mês de janeiro o mais chuvoso (média de $198 \mathrm{~mm}$ ) e o mês de agosto, o mais seco (média de $75 \mathrm{~mm}$ ). A média das temperaturas máximas é de $23,4{ }^{\circ} \mathrm{C}$, a das temperaturas médias, $18,2{ }^{\circ} \mathrm{C}$, e a das mínimas, $13,0^{\circ} \mathrm{C}$ (INMET, 2012).

Os dados meteorológicos (Figura 1) foram obtidos junto ao INMET (Instituto Nacional de Meteorologia), tendo como referência informações diárias de uma 


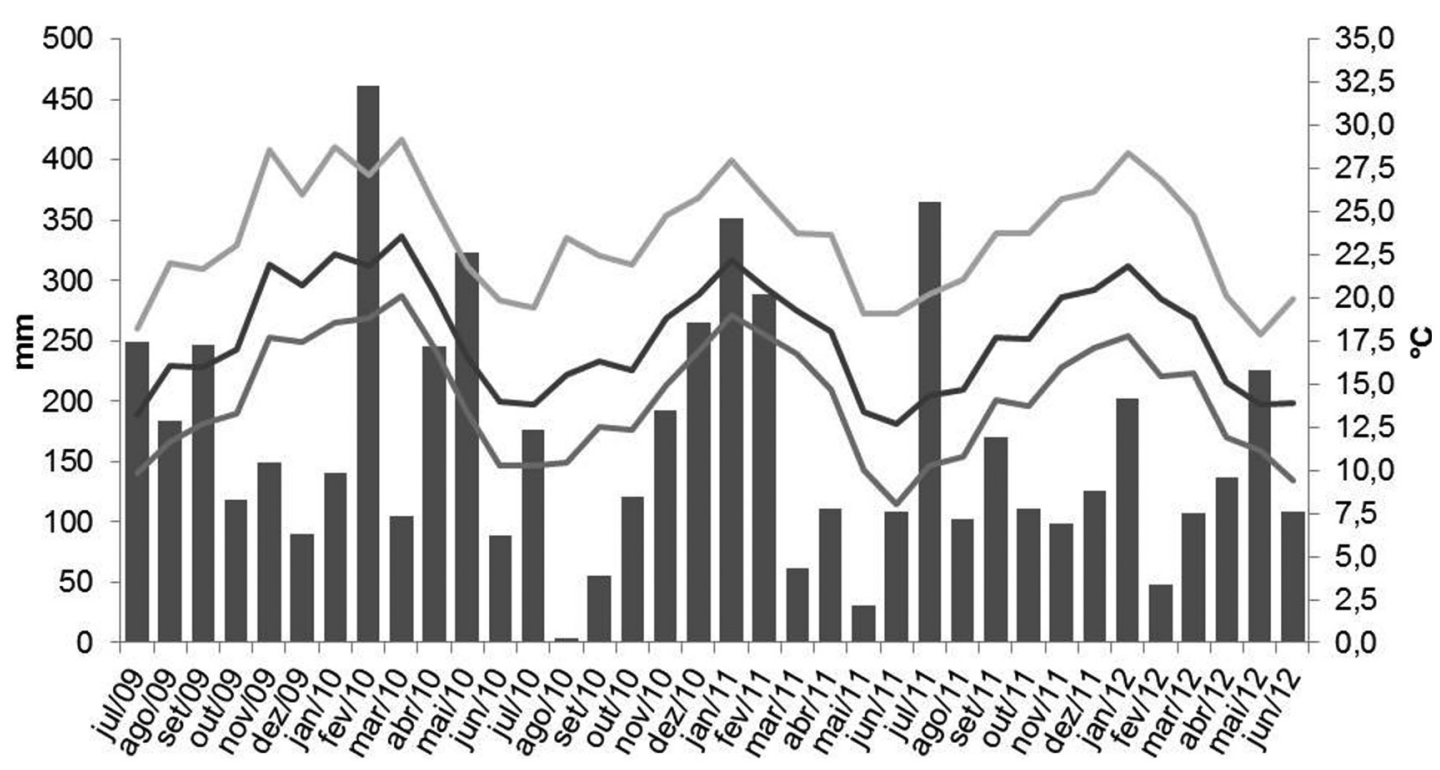

Figura 1. Precipitação (barras) e temperaturas média, mínima e máxima (linhas) em Curitiba, PR, de julho de 2009 a junho de 2012.

Figure 1. Precipitation (bars) and average, minimum and maximum temperatures (lines) at Curitiba-PR, from July 2009 to June 2012.

estação meteorológica da cidade de Curitiba, que dista aproximadamente $20 \mathrm{~km}$ da área estudada, o que permitiu uma melhor correlação entre o incremento em diâmetro das espécies e os dados ambientais.

Para acompanhar a periodicidade e a taxa de incremento em diâmetro das espécies, indivíduos foram selecionados e monitorados por meio de cintas dendrométricas, conforme Fahn et al. (1981), muito empregadas no acompanhamento do crescimento de árvores (e.g.: Botosso et al., 2005; Beedlow et al., 2013; Canetti et al., 2014; Cardoso et al., 2012; Ferreira-Fedele et al., 2004; Figueiredo-Filho et al., 2003; Gomes et al., 2008; Kanieski et al., 2012, 2013; Lisi et al., 2008; Longhi et al., 2006; Silva et al., 2003; Zanon \& Finger, 2010). Esse método consiste em fixar à altura do DAP (diâmetro à altura do peito) cintas permanentes, confeccionadas em aço inoxidável, com precisão de $0,2 \mathrm{~mm}$, o que permite a leitura contínua e precisa dos incrementos em diâmetro. Essas leituras foram realizadas mensalmente por um período de 36 meses, de julho de 2009 a junho de 2012.

Para tanto, foram selecionados, em fase adulta, 44 indivíduos do dossel e nove do sub-bosque de $S$. commersoniana e 8 indivíduos para cada espécie exótica, sendo do dossel $(n=6)$ e sub-bosque $(n=2)$ para L. lucidum e apenas do dossel para H. dulcis.
Para o período de crescimento foram calculados os incrementos correntes mensais médios (ICM) e incrementos correntes anuais (ICA) e, esses, correlacionados com variáveis meteorológicas, precipitação, temperatura média, temperatura mínima e temperatura máxima com o auxílio do aplicativo computacional SPSS (Statistical Package for the Social Sciences). Também foram calculados os incrementos acumulados do tronco ao longo do período e esses foram representados graficamente para melhor comparação entre o incremento das três espécies.

Com a finalidade de verificar as diferenças de crescimento entre S. commersoniana e as espécies exóticas, assim como entre os indivíduos do dossel e os do sub-bosque, foi feita uma Análise de Coordenadas Principais (PCoA) no aplicativo PC-Ord. A PCoA é uma alternativa à Análise de Componentes Principais (PCA), sendo bastante comum em estudos de ecologia por utilizar diversas medidas de distância como Bray-Curtis, Sorensen e Jaccard. O número de coordenadas para a PCoA foi estabelecido pelo Teste de Randomização (999 vezes) e a distância utilizada foi a de Jaccard. Também foi realizada uma análise de variância (ANOVA) por meio do teste de Scott e Knott em nível de 5\% de probabilidade quando houve significância no teste F por meio do aplicativo SISVAR. 


\section{RESULTADOS E DISCUSSÃO}

O incremento em diâmetro foi muito diverso entre as espécies, variando segundo a posição sociológica (dossel ou sub-bosque) e conforme o fato de ser nativa ou invasora exótica. Nota-se que as taxas de incremento acumulado dos indivíduos das espécies exóticas são bem superiores às dos indivíduos das nativas, havendo diferença significativa entre eles. Da mesma forma, as taxas de incremento dos indivíduos do sub-bosque são menores que as dos indivíduos do dossel para cada espécie, havendo também diferença significativa (Figuras 2 e 3).

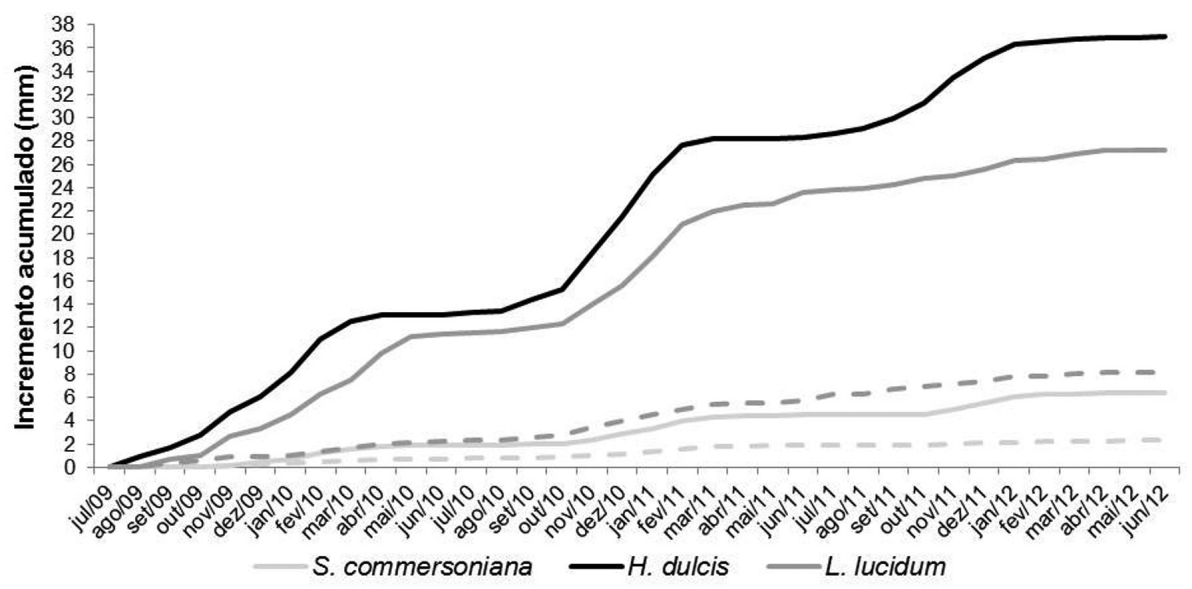

Figura 2. Incremento acumulado em diâmetro do tronco para indivíduos do dossel (linha contínua) e sub-bosque (linha tracejada) das espécies Sebastiania commersoniana, Hovenia dulcis e Ligustrum lucidum, de julho de 2009 a junho de 2012.

Figure 2. Cumulative increase in diameter of the trunk to canopy individuals (solid line) and understory (dashed line) of the species Sebastiania commersoniana, Ligustrum lucidum and Hovenia dulcis from July 2009 to June 2012.

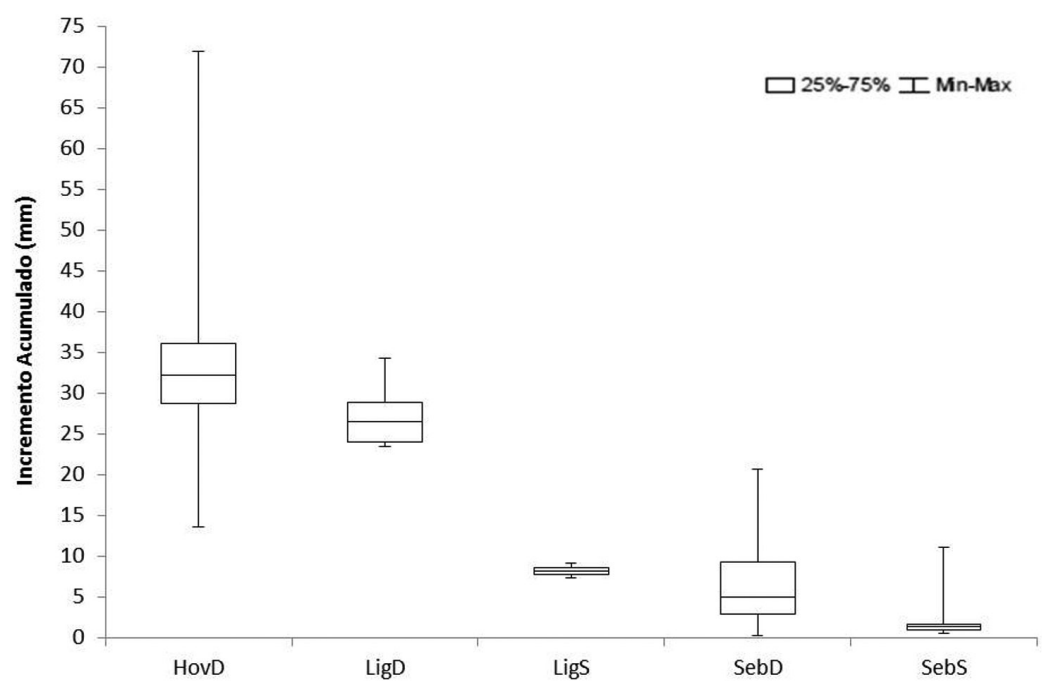

Figura 3. Incremento acumulado em diâmetro do tronco para indivíduos arbóreos em uma área de Floresta Ombrófila Mista Aluvial no período de julho de 2009 a junho de 2012. HovD = Hovenia dulcis do dossel $(\mathrm{n}=8)$; LigD = Ligustrum lucidum do dossel $(\mathrm{n}=6) ;$ LigS = Ligustrum lucidum do sub-bosque; SebD = Sebastiania commersoniana do dossel $(\mathrm{n}=44)$; SebS = Sebastiania commersoniana do sub-bosque $(\mathrm{n}=9)$.

Figure 3. Accumulated increase in trunk diameter for individual trees in an area of Alluvial Mixed Rain Forest from July 2009 to June 2012. HovD = Hovenia dulcis from canopy $(\mathrm{n}=8)$; LigD = Ligustrum lucidum from canopy $(\mathrm{n}=6)$; LigS = Ligustrum lucidum from understory; SebD = Sebastiania commersoniana from canopy $(\mathrm{n}=44)$; SebS = Sebastiania commersoniana from understory $(n=9)$. 
Para as três espécies, o período de maior crescimento em diâmetro, tanto para os indivíduos do sub-bosque quanto para os do dossel, foi de julho de 2010 a junho de 2011. Ao se analisar a Figura 1, referente aos dados meteorológicos, é possível verificar que a precipitação foi maior e mais constante no período em que ocorreu o maior incremento das espécies (de outubro de 2010 a fevereiro de 2011), sendo possivelmente esse o motivo para o maior incremento. A precipitação também pode ter sido o motivo do menor crescimento em diâmetro das espécies no último ano de observação (de julho de 2011 a junho de 2012), em que se nota um baixo índice pluviométrico (Figura 1), principalmente nos meses em que as espécies comumente apresentam maior desempenho.

Ao se analisar o incremento em diâmetro do tronco dos indivíduos de S. commersoniana (Tabela 1), observam-se diferenças significativas entre os indivíduos do dossel e do sub-bosque, mas não houve diferenças significativas entre os anos avaliados. Os indivíduos do dossel iniciam o crescimento em diâmetro no mês de novembro e o mantém até aproximadamente o mês de março. Em abril, o incremento diminui e praticamente estabiliza-se até o mês de outubro. Milani (2013), ao acompanhar o comportamento fenológico das mesmas espécies, observou a existência de dois períodos reprodutivos para $S$. commersoniana de dossel, o primeiro de agosto a novembro e o segundo, de menor intensidade, no mês de fevereiro. Esse primeiro período reprodutivo é iniciado antes da retomada do crescimento em diâmetro da espécie (novembro), podendo, assim, configurar uma boa realocação dos recursos disponíveis tanto para os eventos fenológicos quanto para o incremento diamétrico.

Os indivíduos do sub-bosque de S. commersoniana registraram crescimento bastante baixo, com pouca variação ao longo do ano, apresentando valores de no máximo 0,22 mm de incremento diamétrico nos meses de fevereiro e de março do ano 2012. Gomes et al. (2008), ao avaliarem o crescimento de espécies em uma área de Floresta Ombrófila Mista no Rio Grande do Sul, encontraram resultados semelhantes, com pouca variação no incremento dos indivíduos de S. commersoniana ao longo das diferentes estações, com incremento levemente superior no verão, como seria o esperado.

Hovenia dulcis apresentou crescimento vigoroso ao longo dos meses analisados, com um incremento em diâmetro muito superior às demais espécies analisadas. Embora tenha uma diminuição no incremento no último ano avaliado, não houve diferença significativa entre eles (Tabela 2). A espécie diminui consideravelmente o crescimento em diâmetro do mês de abril até junho, porém retoma o crescimento já no mês de julho, enquanto S. commersoniana o faz apenas depois de novembro. $H$. dulcis apresentou picos de crescimento em diâmetro diferentes ao longo dos três anos, variando entre os meses de novembro, janeiro e fevereiro.

As taxas de crescimento de $H$. dulcis são muito altas, sendo que nos meses de verão o incremento diamétrico mensal da espécie chegou a ser maior que o incremento médio anual de $S$. commersoniana.

Tabela 1. Incrementos correntes mensais em diâmetro $(\mathrm{mm})$ de Sebastiania commersoniana, de julho de 2009 a junho de 2012, em uma Floresta Ombrófila Mista Aluvial, no Paraná.

Table 1. Monthly current increments in diameter $(\mathrm{mm})$ of Sebastiania commersoniana, from July 2009 to June 2012 , in an Alluvial Mixed Rain Forest, PR.

\begin{tabular}{cccccccccccccc} 
Ano & jul & ago & set & out & nov & dez & jan & fev & mar & abr & mai & jun & $\begin{array}{c}\text { ICA } \\
\text { (mm) }\end{array}$ \\
\hline 2009/10 D & 0,00 & 0,02 & 0,02 & 0,02 & 0,13 & 0,27 & 0,21 & 0,52 & 0,33 & 0,22 & 0,13 & 0,02 & $\mathbf{1 , 8 8 a 1}$ \\
\hline $\mathbf{2 0 1 0} / \mathbf{1 1}$ D & 0,05 & 0,01 & 0,04 & 0,02 & 0,31 & 0,53 & 0,52 & 0,64 & 0,30 & 0,08 & 0,02 & 0,09 & $\mathbf{2 , 6 1 a 1}$ \\
\hline $\mathbf{2 0 1 1} / \mathbf{1 2}$ D & 0,04 & 0,01 & 0,02 & 0,00 & 0,42 & 0,53 & 0,57 & 0,17 & 0,08 & 0,04 & 0,03 & 0,02 & $\mathbf{1 , 9 4 a 1}$ \\
\hline $\mathbf{2 0 0 9} / \mathbf{1 0}$ S & 0,00 & 0,01 & 0,01 & 0,04 & 0,09 & 0,11 & 0,13 & 0,12 & 0,08 & 0,10 & 0,03 & 0,01 & $\mathbf{0 , 7 4 a 2}$ \\
\hline $\mathbf{2 0 1 0} / \mathbf{1 1}$ S & 0,08 & 0,01 & 0,01 & 0,10 & 0,10 & 0,11 & 0,20 & 0,22 & 0,22 & 0,03 & 0,04 & 0,04 & $\mathbf{1 , 1 5 a 2}$ \\
\hline $\mathbf{2 0 1 1} / \mathbf{1 2}$ S & 0,01 & 0,02 & 0,01 & 0,00 & 0,12 & 0,11 & 0,01 & 0,05 & 0,03 & 0,01 & 0,03 & 0,01 & $\mathbf{0 , 4 2 a 2}$ \\
\hline ICM D & 0,03 & 0,01 & 0,03 & 0,01 & 0,29 & 0,44 & 0,43 & 0,44 & 0,24 & 0,11 & 0,06 & 0,04 & $\mathbf{2 , 1 4}$ \\
\hline ICM S & 0,03 & 0,02 & 0,01 & 0,04 & 0,10 & 0,11 & 0,12 & 0,13 & 0,11 & 0,05 & 0,03 & 0,02 & $\mathbf{0 , 7 7}$ \\
\hline
\end{tabular}

$\mathrm{D}=$ dossel (44 indivíduos); $\mathrm{S}=$ sub-bosque (9 indivíduos); ICA = Incremento Corrente Anual; ICM = Incremento Corrente Mensal Médio. Letras e números diferentes representam diferença significativa a um nível de 0,05 de probabilidade segundo o teste de Scott e Knott, sendo que as letras referem-se aos anos de incremento e os números, à posição sociológica. 
Os baixos valores de incremento registrados para a espécie nativa, quando comparados a $\mathrm{H}$. dulcis, podem ser, em parte, atribuídos aos dois períodos reprodutivos que ela apresenta (Milani, 2013).

Segundo Rodolfo et al. (2008), essa espécie, que é muito rústica e cresce rapidamente, tem invadido áreas de floresta, onde disputa luz, nutrientes, espaço e fauna dispersora com as nativas. Esse desempenho, em parte, justifica o estabelecimento e a expansão dessa espécie nos ambientes aluviais paranaenses, o que deve representar uma preocupação a mais na manutenção dos poucos remanescentes minimamente conservados da região.

Ligustrum lucidum apresentou crescimento diferenciado de acordo com a posição sociológica dos indivíduos, tendo de forma geral, os do dossel, crescimento diamétrico mais expressivo que os do sub-bosque, embora não tenha havido diferença significativa (Tabela 3). O menor incremento dos indivíduos do sub-bosque é justificado pelo fato de a espécie ser pioneira, ter características de rápido crescimento e dominar o dossel da floresta. Os indivíduos do sub-bosque, que não encontram essas condições, acabam tendo seu crescimento bastante prejudicado.

Ligustrum lucidum diminui seu crescimento em diâmetro também nos meses mais frios, principalmente maio a agosto, e em setembro o retoma. A espécie tem suas fenofases vegetativas de renovação de folhas ocorrendo de forma contínua, o que pode ser entendido como uma estratégia para garantir seu sucesso quanto à ciclagem de nutrientes. A espécie passa praticamente seis meses (setembro a março) expressando suas fenofases reprodutivas (Milani, 2013). Esse período é compatível com o início do maior incremento em diâmetro da espécie.

No último ano de observação, os indivíduos do dossel de L. lucidum diminuíram bastante o incremento, assim como observado nas outras espécies, obtendo

Tabela 2. Incrementos correntes mensais em diâmetro (mm) de Hovenia dulcis, de julho de 2009 a junho de 2012, em uma Floresta Ombrófila Mista Aluvial, no Paraná.

Table 2. Monthly current increments in diameter (mm) of Hovenia dulcis, from July 2009 to June 2012, in an Alluvial Mixed Rain Forest, PR.

\begin{tabular}{|c|c|c|c|c|c|c|c|c|c|c|c|c|c|}
\hline Ano & jul & ago & set & out & nov & dez & jan & fev & mar & abr & mai & jun & $\begin{array}{c}\text { ICA } \\
(\mathbf{m m})\end{array}$ \\
\hline $2009 / 10 \mathrm{D}$ & 0,00 & 0,94 & 0,69 & 1,19 & 1,98 & 1,32 & 2,02 & 2,81 & 1,62 & 0,47 & 0,05 & 0,00 & $13,10 \mathrm{a}$ \\
\hline 2010/11 D & 0,24 & 0,08 & 1,00 & 0,90 & 3,03 & 3,21 & 3,55 & 2,58 & 0,48 & 0,05 & 0,04 & 0,03 & $15,19 a$ \\
\hline $2011 / 12 \mathrm{D}$ & 0,35 & 0,44 & 0,95 & 1,25 & 2,24 & 1,66 & 1,20 & 0,20 & 0,20 & 0,06 & 0,08 & 0,03 & $8,67 a$ \\
\hline ICM D & 0,20 & 0,49 & 0,88 & 1,11 & 2,42 & 2,07 & 2,26 & 1,86 & 0,77 & 0,19 & 0,06 & 0,02 & 12,32 \\
\hline
\end{tabular}

$\mathrm{D}=$ dossel (8 indivíduos); ICA = Incremento Corrente Anual; ICM = Incremento Corrente Mensal Médio. Letras diferentes representam diferença significativa a um nível de 0,05 de probabilidade segundo o teste de Scott e Knott.

Tabela 3. Incrementos correntes mensais em diâmetro ( $\mathrm{mm}$ ) de Ligustrum lucidum, de julho de 2009 a junho de 2012, em uma Floresta Ombrófila Mista Aluvial, no Paraná.

Table 3. Monthly current increments in diameter (mm) of Ligustrum lucidum, from July 2009 to June 2012, in an Alluvial Mixed Rain Forest, PR.

\begin{tabular}{|cccccccccccccc} 
Ano & jul & ago & set & out & nov & dez & jan & fev & mar & abr & mai & jun & $\begin{array}{c}\text { ICA } \\
\text { (mm) }\end{array}$ \\
\hline 2009/10 D & 0,00 & 0,05 & 0,62 & 0,40 & 1,55 & 0,73 & 1,17 & 1,75 & 1,21 & 2,36 & 1,40 & 0,19 & $\mathbf{1 1 , 4 3 a 1}$ \\
\hline 2010/11 D & 0,14 & 0,08 & 0,31 & 0,37 & 1,68 & 1,62 & 2,53 & 2,74 & 1,05 & 0,52 & 0,18 & 0,92 & $\mathbf{1 2 , 1 4 a 1}$ \\
\hline $\mathbf{2 0 1 1} / \mathbf{1 2}$ D & 0,23 & 0,16 & 0,27 & 0,55 & 0,25 & 0,51 & 0,77 & 0,17 & 0,40 & 0,30 & 0,02 & 0,03 & $\mathbf{3 , 6 7 a 1}$ \\
\hline $\mathbf{2 0 0 9 / 1 0 ~ S ~}$ & 0,00 & 0,03 & 0,38 & 0,13 & 0,38 & 0,03 & 0,06 & 0,38 & 0,25 & 0,35 & 0,16 & 0,10 & $\mathbf{2 , 2 6 a 1}$ \\
\hline $\mathbf{2 0 1 0} / \mathbf{1 1}$ S & 0,10 & 0,00 & 0,19 & 0,25 & 0,60 & 0,62 & 0,47 & 0,51 & 0,41 & 0,13 & 0,03 & 0,16 & $\mathbf{3 , 4 7 a 1}$ \\
\hline $\mathbf{2 0 1 1 / 1 2 ~ S ~}$ & 0,54 & 0,03 & 0,38 & 0,29 & 0,16 & 0,29 & 0,38 & 0,03 & 0,19 & 0,13 & 0,03 & 0,03 & $\mathbf{2 , 4 8 a 1}$ \\
\hline ICM D & 0,12 & 0,10 & 0,40 & 0,44 & 1,16 & 0,95 & 1,49 & 1,55 & 0,89 & 1,06 & 0,53 & 0,38 & $\mathbf{9 , 0 8}$ \\
\hline ICM S & 0,21 & 0,02 & 0,32 & 0,22 & 0,38 & 0,31 & 0,30 & 0,31 & 0,29 & 0,20 & 0,07 & 0,10 & $\mathbf{2 , 7 4}$ \\
\hline
\end{tabular}

$\mathrm{D}=$ dossel (6 indivíduos); $\mathrm{S}$ = sub-bosque (2 indivíduos); ICA = Incremento Corrente Anual; ICM = Incremento Corrente Mensal Médio. Letras e números diferentes representam diferença significativa a um nível de 0,05 de probabilidade segundo o teste de Scott e Knott, sendo que as letras referem-se aos anos de incremento e os números, à posição sociológica. 
valores semelhantes aos do sub-bosque. O crescimento menor possivelmente está relacionado a uma menor precipitação nesse período, sendo que a espécie respondeu de maneira mais evidente a essa variável ambiental.

Os resultados da Análise de Coordenadas Principais mostram claramente um grupo formado pelas espécies exóticas (Figura 4) e um grupo formado pelos indivíduos do sub-bosque (Figura 5), sendo que a primeira coordenada explica $18,51 \%$ da variação dos dados e a segunda, $13,65 \%$.

No grupo das espécies exóticas nota-se apenas dois indivíduos que não estão agrupados com os demais. Esses indivíduos da espécie L. lucidum são os únicos que estão localizados no sub-bosque da floresta. Essa condição de menor luminosidade no interior da floresta faz com que o crescimento desses indivíduos acabe diferenciando-se do padrão de crescimento diamétrico dos demais. Esses mesmos indivíduos estão distantes do agrupamento dos de sub-bosque. Isso se deve ao fato de que, mesmo crescendo menos que os indivíduos de L. lucidum do dossel, eles têm um desempenho superior aos indivíduos do sub-bosque da espécie nativa.

No grupo do sub-bosque, um dos indivíduos de S. commersoniana mostrou um comportamento diferente do seu grupo, assemelhando-se ao crescimento dos do dossel. Esse crescimento diferenciado pode estar ocorrendo por diversos fatores, como menor competição e consequente maior disponibilidade de recursos, características genéticas da planta e, como citado por Longhi-Santos (2013), pode indicar que o indivíduo provém de rebrota, o que faz que ele tenha um sistema radicial consistente e que parte significativa dos recursos obtidos seja direcionada para o crescimento aéreo.

Na Tabela 4 podem ser observadas as correlações entre o crescimento das espécies e as variáveis meteorológicas testadas. Foram obtidas correlações significativas para todas as espécies, com praticamente todas as variáveis, principalmente temperatura.

De acordo com Husch et al. (1982), o crescimento em diâmetro das árvores é influenciado pelas características

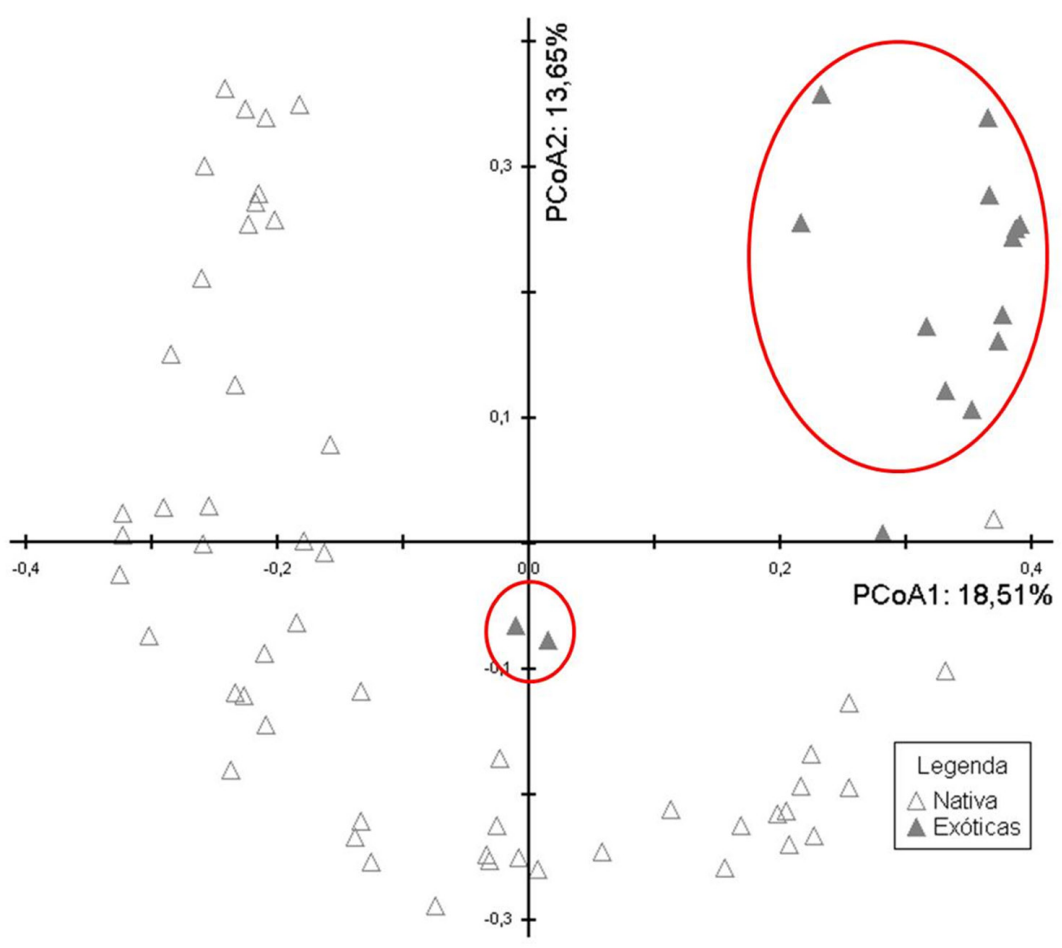

Figura 4. Análise das coordenadas principais para incremento corrente mensal (ICM) em diâmetro do tronco entre o grupo da espécie nativa e o das espécies exóticas invasoras.

Figure 4. Analysis of the main coordinates for monthly current increment (ICM) in trunk diameter between the native species group and the group of invasive exotic species. 


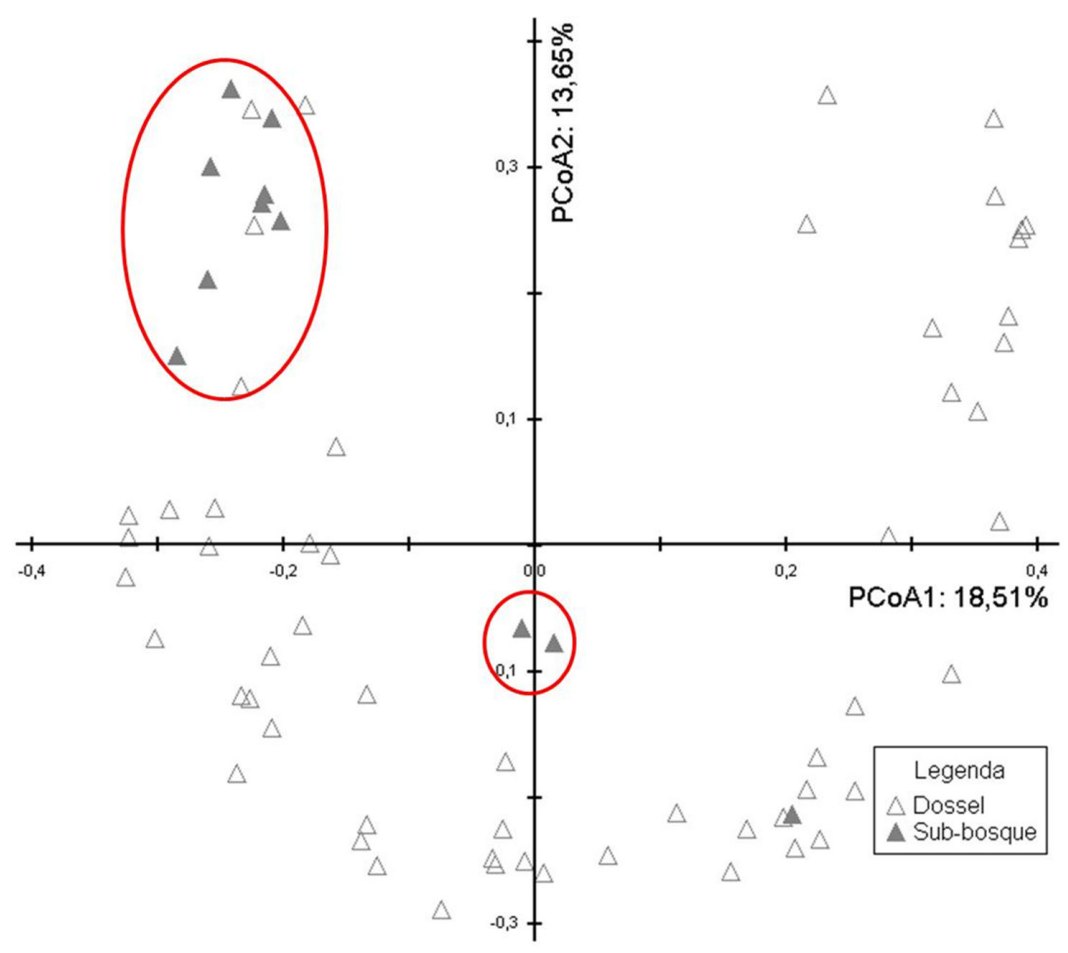

Figura 5. Análise das coordenadas principais para incremento corrente mensal (ICM) em diâmetro do tronco entre o grupo dos indivíduos do dossel e o dos indivíduos do sub-bosque.

Figure 5. Analysis of the main coordinates for monthly current increment (ICM) in trunk diameter between the group of canopy individuals and the understory individuals.

Tabela 4. Correlações entre o incremento corrente mensal das espécies e as variáveis meteorológicas.

Table 4. Correlations between monthly current increment and weather variables.

\begin{tabular}{lccccc} 
Variáveis meteorológicas & \multicolumn{5}{c}{ Espécies } \\
\cline { 2 - 6 } & SD & SS & H & LD & LS \\
Precipitação & $0,393^{*}$ & 0,223 & $0,410^{*}$ & $0,534^{* *}$ & $0,540^{* *}$ \\
Temperatura máxima & $0,697^{* *}$ & $0,529^{* *}$ & $0,693^{* *}$ & $0,582^{* *}$ & $0,414^{*}$ \\
Temperatura mínima & $0,769^{* *}$ & $0,659^{* *}$ & $0,706^{* *}$ & $0,675^{* *}$ & $0,480^{* *}$ \\
Temperatura média & $0,754^{* *}$ & $0,614^{* *}$ & $0,714^{* *}$ & $0,645^{* *}$ & $0,453^{* *}$ \\
\hline
\end{tabular}

$\mathrm{SD}=$ Sebastiania commersoniana do dossel; $\mathrm{SS}=$ Sebastiania commersoniana do sub-bosque; $\mathrm{H}=$ Hovenia dulcis; $\mathrm{LD}=$ Ligustrum lucidum do dossel; LS = Ligustrum lucidum do sub-bosque. ${ }^{*}$ Correlação de Pearson significativa a um nível de 0,$05 ;{ }^{* *}$ Correlação significativa a um nível de 0,01 .

da espécie interagindo com o ambiente, inclusive fatores meteorológicos. Segundo Cardoso (1991), a temperatura é considerada um dos principais fatores para a ativação do câmbio. Dünisch (2005) relata que em áreas de clima tropical, nas quais há uma estacionalidade climática definida, o crescimento vegetal é influenciado principalmente pela precipitação, e que, em áreas de clima subtropical, a temperatura exerce maior influência sobre o crescimento.

A temperatura atua diretamente sobre a iniciação e duração da atividade cambial. Geralmente o câmbio é ativado depois de uma temperatura crítica mínima ser alcançada, então a atividade cambial progride com o aumento da temperatura até chegar a um nível superior crítico, a partir do qual a atividade do câmbio começa a declinar (Went, 1953).

Zanon \& Finger (2010), em um estudo da relação entre variáveis meteorológicas e o crescimento em diâmetro de Araucaria angustifolia (Bertol.) Kuntze, na Floresta Nacional de São Francisco de Paula, RS, verificaram que o aumento da temperatura correspondia a maior crescimento, que cessava ou reduzia-se nos 
períodos de temperaturas mais baixas, ocasiões em que também ocorria excesso de água no solo.

Figueiredo-Filho et al. (2008), ao avaliarem o incremento diamétrico de sete espécies de uma área de Floresta Ombrófila Mista no sul do Paraná, encontraram a correlação desse principalmente com a temperatura, pelo fato de a temperatura ter um padrão similar ao obtido no incremento, com maiores valores no verão e menores no inverno, sendo que a precipitação nesse caso não teve correlação significativa por apresentar um padrão de distribuição irregular ao longo do ano.

Segundo Carlquist \& Hoekman (1985), a água, quer em excesso quer em escassez, é um dos principais fatores que afetam a atividade cambial. A falta de água ocasiona a interrupção do alongamento dos ramos jovens, levando a um decréscimo no suprimento de Ácido Indol Acético (AIA), que é um importante estimulador do câmbio. Já o excesso de água gera um decréscimo do potencial osmótico, o que estimula uma grande diferenciação de xilema e consequente menor crescimento.

Analisando a correlação do crescimento em diâmetro das espécies com a precipitação, apenas os indivíduos de $S$. commersoniana do sub-bosque não apresentaram correlação significativa, possivelmente por terem melhores condições de umidade no interior da floresta, o que gera também menores perdas de água por transpiração e respiração, não sendo a precipitação um fator tão condicionante para o crescimento nessa situação.

Ligustrum lucidum foi a espécie com maior correlação com precipitação, tanto para indivíduos do sub-bosque, quanto para os do dossel. Essa maior correlação com a precipitação auxilia na explicação do incremento consideravelmente menor da espécie no último ano estudado, o qual teve índices pluviométricos menores.

Para S. commersoniana, a maior correlação foi com a temperatura mínima, assim como para L. lucidum. Essa alta correlação com a temperatura mínima deve se dar principalmente no período de maior crescimento e de temperaturas mais altas, temperaturas mais amenas auxiliam na diminuição de perdas de água por evapotranspiração, convertendo esse material para aumento de biomassa.

Para $H$. dulcis, a maior correlação foi com a temperatura média, o que mostra que o ambiente proporciona condições ideais para o desenvolvimento e estabelecimento da espécie, justificando os altos valores de incremento diamétrico apresentados.

\section{CONCLUSÕES}

Com base nos resultados obtidos, é possível concluir que:

- As espécies apresentaram incremento diamétrico distinto entre elas e em relação à posição sociológica;

- O incremento diamétrico é influenciado pelas condições meteorológicas, observando-se em Sebastiania commersoniana correlações significativas com temperatura e, em Hovenia dulcis e Ligustrum lucidum, tanto com a temperatura quanto com a precipitação;

- As informações geradas neste estudo podem fornecer subsídios para futuros projetos que possam diminuir a degradação em áreas similares no Sul do Brasil, a partir do conhecimento dos padrões de crescimento das espécies avaliadas.

\section{AGRADECIMENTOS}

Os autores agradecem à CAPES e ao CNPQ pelo auxílio financeiro.

\section{STATUS DA SUBMISSÃO}

Recebido: 4 dez., 2014

Aceito: 15 maio, 2016

AUTOR(ES) PARA CORRESPONDÊNCIA:

\section{Maria Raquel Kanieski}

Departamento de Engenharia Florestal, Universidade do Estado de Santa Catarina UDESC, Avenida Luiz de Camões, 2090, CEP 88520-000, Lages, SC, Brasil e-mail: raquelkanieski@gmail.com

\section{REFERÊNCIAS}

Andreacci F, Botosso PC, Galvao F. Sinais climáticos em anéis de crescimento de Cedrela fissilis em diferentes tipologias de florestas ombrófilas do sul do Brasil. Floresta 2014; 44(2): 323-332. http://dx.doi.org/10.5380/rf.v44i2.27316.

Beedlow PA, Lee EH, Tingey DT, Waschmann RS, Burdick $\mathrm{CA}$. The importance of seasonal temperature and moisture patterns on growth of Douglas-fir in western Oregon, US. Agricultural and Forest Meteorology 2013; 169: 174-185. http://dx.doi.org/10.1016/j.agrformet.2012.10.010. 
Botosso PC, Tomazello M Fo, Maria VRB, Ferreira-Fedele L. Les lianes et l'accroissement diametral dês arbres de Centrolobium tomentosum Guill. Ex Benth. (Caesalpinaceae) au Brésil. Bois et Forêts des Tropiques 2005; 284(2): 71-75.

Brienen RJW, Zuidema PA. Lifetime growth patterns na ages of Bolivian rain forests trees obtained by tree ring analysis. Journal of Ecology 2006; 94(2): 481-493. http:// dx.doi.org/10.1111/j.1365-2745.2005.01080.x.

Canetti A, Ruy CC, Mattos PP, Braz EM. Dinâmica de crescimento de espécies de um remanescente de Floresta Ombrófila Mista em Colombo, PR. Pesquisa Florestal Brasileira 2014; 34(77): 31-37. http://dx.doi. org/10.4336/2014.pfb.34.77.602.

Cardoso FCG, Marques R, Botosso PC, Marques MCM. Stem growth and phenology of two tropical trees in contrasting soil conditions. Plant and Soil 2012; 354(1-2): 269-281. http://dx.doi.org/10.1007/s11104-011-1063-9.

Cardoso NS. Caracterização da estrutura anatômica da madeira, fenologia e relações com a atividade cambial de árvores de teca (Tectona grandis) - Verbanaceae [dissertação]. Piracicaba: Escola Superior de Agricultura "Luiz de Queiroz", Universidade de São Paulo; 1991.

Carlquist S, Hoekman DA. Ecological wood anatomy of the woody Southern Californian flora. Iawa Bulletin 1985; 6(4): 319-347. http://dx.doi.org/10.1163/22941932-90000960.

Dünisch O. Influence of the El-Niño southern oscillation on cambial growth of Cedrela fissilis Vell in tropical and subtropical Brazil. Journal of Applied Botany and Food Quality 2005; 79: 5-11.

Fahn A, Burley J, Longman KA, Mariaux A, Tonlinson PB. Possible contributions of wood anatomy to the determination of the age of tropical trees. In: Bormann FH, Berlyn G. Age and growth rate of tropical trees: new directions for research. New Haven: Yale University; 1981. p. 31-54.

Ferreira-Fedele L, Tomazello M Fo, Botosso PC, Giannotti E. Periodicidade do crescimento de Esenbeckia leiocarpa Engl. (guarantã) em duas áreas da região Sudeste do Estado de São Paulo. Scientia Florestalis 2004; 65(65): 141-149.

Figueiredo-Filho A, Hubie SR, Schaaf LB, Figueiredo DJ, Sanquetta CR. Avaliação do incremento em diâmetro com o uso de cintas dendrométricas em algumas espécies de uma Floresta Ombrófila Mista localizada no Sul do Estado do Paraná. Revista Ciências Exatas e Naturais 2003; 5(1): 69-84.

Figueiredo-Filho A, Rode R, Figueiredo DJ, Machado S. do A. Seasonal diameter increment for 7 species from an Ombrophyllous Mixed Forest, Southern state of Paraná, Brazil. Floresta 2008; 38(3): 527-543. http://dx.doi. org/10.5380/rf.v38i3.12424.

Gomes JF, Longhi SJ, Araujo MM, Brena DA. Classificação e crescimento de unidades de vegetação em Floresta Ombrófila Mista, São Francisco de Paula, RS. Ciência Florestal 2008; 18(1): 93-107. http://dx.doi.org/10.5902/19805098514.
Husch B, Miller CI, Beers TW. Forest mensuration. 3. ed. New York: John Wiley \& Sons; 1982. 402 p.

Instituto Nacional de Meteorologia - INMET. Banco de dados meteorológicos para ensino e pesquisa [online]. 2012 [citado em 2012 dez 10]. Disponível em: http:// www.inmet.gov.br/portal/index.php? $r=b d m e p / b d m e p$

Kanieski MR, Longhi-Santos TL, Graf J No, Souza T, Galvão F, Roderjan CV. Influência da precipitação e da temperatura no incremento diamétrico de espécies florestais aluviais em Araucária-PR. Floresta e Ambiente 2012; 19(1): 17-25. http://dx.doi.org/10.4322/floram.2012.003.

Kanieski MR, Longhi-Santos TL, Milani JEF, Miranda BP, Galvão F, Botosso PC et al. Crescimento Diamétrico de Blepharocalyx salicifolius em Remanescente de Floresta Ombrófila Mista Aluvial, Paraná. Floresta e Ambiente 2013; 20(2): 197-206. http://dx.doi.org/10.4322/floram.2013.007.

Lisi CS, Tomazello-Filho M, Botosso PC, Roig FA, Maria VRB, Ferreira-Fedele L et al. Tree-ring formation, radial increment periodicity and phenology of tree species from a Seasonal Semi-Decidous Forest in Southeast Brazil. IAWA Journal 2008; 29(2): 189-207. http://dx.doi. org/10.1163/22941932-90000179.

Longhi SJL, Brena DA, Gomes JF, Narvaes IS, Berger G, Soligo AJ. Classificação e caracterização de estágios sucessionais em remanescentes de Floresta Ombrófila Mista na FLONA de São Francisco de Paula, RS, Brasil. Ciência Florestal 2006; 16(2): 113-125. http://dx.doi. org/10.5902/198050981893.

Longhi-Santos T. Dendroecologia de Sebastiania commersoniana (Baill.) L.B.Sm. \& Downs em um fragmento de Floresta Ombrófila Mista Aluvial, Paraná, Brasil [dissertação]. Curitiba: Setor de Ciências Agrárias, Universidade Federal do Paraná; 2013.

Milani JEF. Comportamento fenológico de espécies arbóreas em um fragmento de Floresta Ombrófila Mista Aluvial [dissertação]. Curitiba: Setor de Ciências Agrárias, Universidade Federal do Paraná; 2013.

Nutto L, Watzlawick LF. Relações entre fatores climáticos e incremento em diâmetro de Zanthoxyllum rhoifolia Lam.e Zanthoxyllum hyemale St. Hil. na região de Santa Maria, RS. Boletim de Pesquisa Florestal 2002; 45: 41-55.

Paraná. Instituto Ambiental do Paraná. Portaria IAP $n^{\circ}$ 074, de 22 de maio de 2007. Reconhece a Lista Oficial de Espécies Exóticas Invasoras para o estado do Paraná, estabelece normas de controle e dá outras providências. Curitiba (2007 maio 22): 8.

Rodolfo AL, Cândido JF Jr, Temponi LG, Gregorini MZ. Citrus aurantium L. (laranja-apepu) e Hovenia dulcis Thunb. (uva-do-Japão): espécies exóticas invasoras da trilha do Poço Preto no Parque Nacional do Iguaçu, Paraná, Brasil. Revista Brasileira de Biociências 2008; 6(1): 16-18.

Silva RP, Nakamura S, Azevedo CP, Chambers J, Rocha $\mathrm{RM}$, Pinto ACM et al. Uso de banda dendrométrica na 
definição de padrões de crescimento individual em diâmetro de árvores da bacia do rio Cuieiras. Acta Amazonica 2003; 33(1): 67-84. http://dx.doi.org/10.1590/1809-4392200331084.

Socher LG, Roderjan CV, Galvão F. Biomassa aérea de uma Floresta Ombrófila Mista Aluvial no município de Araucária (PR). Floresta 2008; 38(2): 245-252. http:// dx.doi.org/10.5380/rf.v38i2.11619.

Spathelf P, Fleig FD, Vaccaro S, Esber LM. Análise dendroecológica de Ocotea pulchella Nees et Mart. ex
Nees (canela-lageana) na Serra Geral de Santa Maria, RS, Brasil. Ciência Florestal 2000; 10(1): 95-108.

Went FW. The effect of temperature on plant growth. Annual Review of Plant Physiology 1953; 4(1): 347-362. http://dx.doi.org/10.1146/annurev.pp.04.060153.002023. Zanon MLB, Finger CAG. Relação de variáveis meteorológicas com o crescimento das árvores de Araucaria angustifolia (Bertol.) Kuntze em povoamentos implantados. Ciência Florestal 2010; 20(3): 467-476. http:// dx.doi.org/10.5902/198050982061. 